\title{
AVALIAÇÃO DA QUALIDADE DE VIDA DE PACIENTES \\ DIAGNOSTICADOS COM CÂNCER DE BOCA, FARINGE E LARINGE ATENDIDOS NA UNIDADE DE ALTA COMPLEXIDADE EM ONCOLOGIA (UNACON) E MULTICLIN DE FEIRA DE SANTANA - BA
}

\section{Maria Janaína Ramos Almeida; Márcio Campos Oliveira}

\author{
1. Bolsista PIBIC/CNPq, Graduando em Odontologia, Universidade Estadual de Feira de Santana, e-mail: \\ mariajanainaa2@outlook.com \\ 2. Orientador, Departamento de saúde, Universidade Estadual de Feira de Santana, e-mail: \\ marciopatologiaoral@gmail.com
}

\begin{abstract}
PALAVRAS-CHAVE: Qualidade de vida; Neoplasias de Cabeça e Pescoço; Inquéritos e Questionários.
\end{abstract}

\section{INTRODUÇÃO}

Câncer é o nome geral dado a um conjunto de mais de 100 doenças, caracterizadas pelo crescimento desordenado de células, que tendem a invadir tecidos e órgãos vizinhos (BRASIL, 2011). Os cânceres de boca, faringe e laringe estão incluídos no grupo de tumores malignos localizados no trato aerodigestivo superior, conhecidos como cânceres de cabeça e pescoço (BRASIL, 2017). Esses tumores malignos resultam da interação de fatores extrínsecos, intrínsecos e herança genética, tratando-se, portanto, de uma doença multifatorial, que afeta preferencialmente os homens, com mais de quarenta anos, etilistas crônicos, fumantes e expostos à radiação solar (BRASIL, 2017; NEVILLE, 2016).

O problema do câncer no Brasil ganha relevância pelo perfil epidemiológico que essa doença vem apresentando. As estimativas do Instituto Nacional do Câncer (INCA) para o biênio 2018-2019 apontam a ocorrência de 600 mil casos novos para cada ano, sendo esperada a ocorrência de 11.200 casos novos de câncer da cavidade oral em homens e 3.500 em mulheres, e 6.390 casos novos de câncer de laringe em homens e 1.280 em mulheres (BRASIL, 2017).

O tumor maligno de cabeça e pescoço é uma doença mutiladora que pode afetar a saúde geral e mental, a aparência, emprego, vida social e vida em família. Também podem ocorrer sérias mudanças no funcionamento do trato aerodigestivo superior com impacto negativo na qualidade de vida do paciente oncológico (GALBIATTI, 2013).

O conceito de qualidade de vida é abrangente, reflete a saúde subjetiva percebida pelo paciente e envolve saúde física, estado psicológico e relações sociais (LOPEZ, 2013). Informações sobre a qualidade de vida podem ser obtidas por meio de entrevistas semiestruturadas e avaliações qualitativas, mas a aplicação de questionários vem sendo o recurso mais utilizado, devido ao baixo custo e facilidade na análise dos resultados (RINGASH, 2001). O questionário escolhido para instrumento de pesquisa do presente estudo foi o UW-QOL (4a versão).

A avaliação da qualidade de vida do paciente oncológico por meio da aplicação de questionários representa um instrumento útil na percepção do impacto físico e psicossocial das enfermidades e eficácia dos diferentes tratamentos, pois pode fornecer informações importantes para auxiliar na conduta de cada caso, possibilitando aos profissionais de saúde a compreensão de como os pacientes vivenciam a evolução da 
doença e as consequências do seu tratamento, com o objetivo de contribuir para a melhoria da prestação de serviços (LIMA, 2011; ALGAVE, 2015).

Diante do exposto, o presente estudo tem como objetivo avaliar a qualidade de vida de pacientes com câncer de boca, faringe e laringe em terapia oncológica na Unidade de Alta Complexidade em Oncologia (UNACON) e Multiclin de Feira de Santana - BA.

\section{METODOLOGIA}

Trata-se de um estudo transversal de caráter descritivo. A pesquisa foi realizada a partir da aplicação do questionário de avaliação de qualidade de vida da Universidade de Washington (UW-QOL) (versão 4) em pacientes diagnosticados com câncer de boca, faringe e laringe antes de iniciarem o tratamento na Unidade de Alta Complexidade em Oncologia (UNACON) e na Multiclin de Feira de Santana - BA, no período de agosto de 2017 a julho de 2018.

O projeto de pesquisa foi aprovado pelo Comitê de Ética em Pesquisa em Seres Humanos (CEP) da Universidade Estadual de Feira de Santana (UEFS), com protocolo $n^{\circ} 1.621 .470$, conforme a Resolução 466/12 que diz respeito à pesquisa envolvendo seres humanos.

Os critérios de inclusão foram: todos os pacientes diagnosticados com câncer de boca, faringe e laringe antes de iniciar o tratamento, com idade igual ou superior a 18 anos de idade e que desejarem participar do estudo. Foram eliminados sujeitos com outros diagnósticos e/ou outros cânceres, aqueles que tinham algum déficit mental, que pudesse dificultar a compreensão das perguntas do questionário, indivíduos que já estavam em tratamento oncológico e aqueles que não aceitaram participar.

O procedimento de coleta de dados foi iniciado com um levantamento dos pacientes que foram diagnosticados com câncer de boca, faringe e laringe e que atenderam aos critérios de inclusão da pesquisa. Em seguida, aplicou-se o questionário de avaliação de qualidade de vida da Universidade de Washington (UW-QOL) (versão 4). Este questionário é composto por doze questões de múltipla escolha, cada questão apresenta de três a cinco categorias de resposta com escore variando de zero (pior) a 100 (melhor). Apresenta também uma questão que permite ao paciente classificar quais problemas são os mais importantes para ele, três questões gerais sobre sua qualidade de vida global e relacionada à saúde e uma questão aberta para os pacientes fazerem seus comentários.

Para análise dos dados utilizou-se o software Statistical Package for Social Science (SPSS), versão 10.0. Posteriormente, as informações foram sistematizadas em tabelas com o auxílio do Programa Excel da MICROSOFT CORPORATION (2007).

\section{RESULTADOS E DISCUSSÃO}

A pesquisa contou com a participação de 44 pacientes. Como se observa na tabela 1, mais da metade dos doentes tiveram o tumor primário localizado em boca (54,5\%). Resultados similares foram encontrados no estudo de Sommerfeld et al (2012), onde dos 9 sítios avaliados a maior proporção dos casos se concentrou na cavidade oral (37,86\%). A média de idade foi de 61,19 anos.

Tabela 1. Localização do tumor primário

\begin{tabular}{r|ll}
\multicolumn{2}{c}{$\boldsymbol{N}$} & \multicolumn{1}{c}{$\%$} \\
\hline Boca & 24 & 54,5 \\
Faringe & 9 & 20,5 \\
Laringe & 11 & 25,0 \\
TOTAL & 44 & 100,0
\end{tabular}


A partir da análise das tabelas 2 e 3 nota-se a maior prevalência no sexo masculino $(75,0 \%)$, provenientes da zona rural $(54,4 \%)$, analfabetos $(45,5 \%)$ e com renda familiar mensal de um salário mínimo (40,9\%). A baixa escolaridade dos pacientes e reduzido poder aquisitivo provavelmente dificulta o acesso aos serviços de saúde para diagnóstico precoce da doença e influencia no entendimento de como será realizado o tratamento, podendo acarretar em falta de colaboração e diminuição do sucesso terapêutico (ANGELO, 2010; OLIVEIRA, 2017).

Tabela 2. Caracterização dos pacientes de acordo com sexo e local de moradia.

\begin{tabular}{c|cc} 
& $\boldsymbol{N}$ & $\mathbf{\%}$ \\
\hline Sexo & & \\
Masculino & 33 & 75,0 \\
Feminino & 11 & 25,0 \\
TOTAL & 44 & 100,0 \\
Local de moradia & & \\
Zona urbana & 20 & 45,5 \\
Zona rural & 24 & 54,4 \\
TOTAL & 44 & 100,0
\end{tabular}

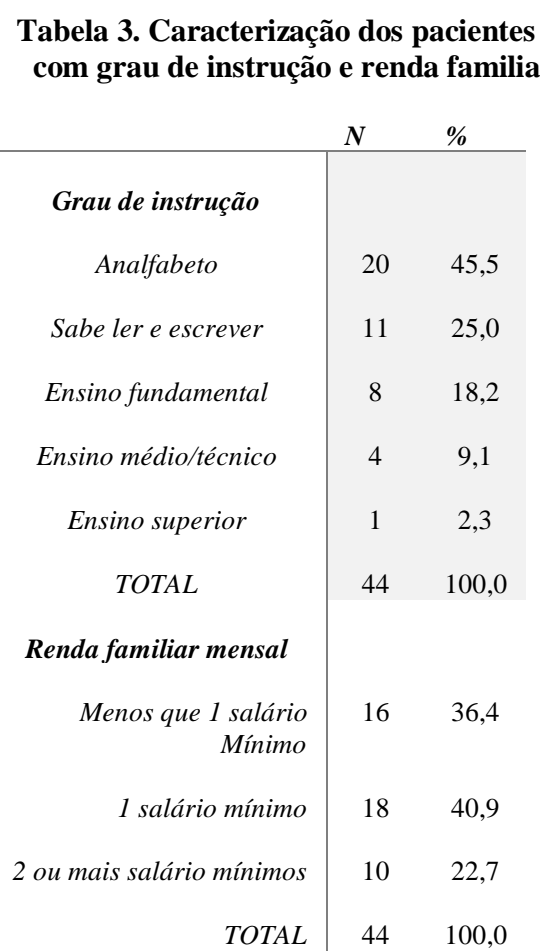

Na tabela 4 está representada a avaliação da qualidade de vida, a partir dos resultados obtidos com a aplicação do questionário de UW-QOL (4 ${ }^{\mathrm{a}}$ versão). Para o câncer de boca os domínios mais afetados foram: mastigação, dor e ansiedade. A mastigação apresentou o pior score $(35,42 \%)$, concordando com os resultados encontrados nos estudos de Angelo (2010) e Sommerfeld (2012). Já no câncer de faringe os mais afetados foram: mastigação, humor, ansiedade e deglutição e no câncer de laringe as menores pontuações foram nos domínios de fala/recreação, paladar e atividade. $\mathrm{O}$ único domínio que apresentou diferença estatisticamente significativa entre os três grupos foi a mastigação, com $\mathrm{p}=0,005$.

A partir da avaliação da pontuação total pode-se observar que a pior qualidade de vida no momento da aplicação do questionário foi nos indivíduos com câncer de boca $(796,29)$ e a melhor nos portadores de câncer de laringe $(900,27)$.

Diante dos resultados expostos, conclui-se que o paciente com câncer de cabeça e pescoço apresenta redução na qualidade de vida, influenciada por fatores socioeconômicos e localização do tumor primário, sendo que os piores valores foram obtidos para o câncer de boca, em indivíduos analfabetos, residentes da zona rural e de baixo poder aquisitivo e os melhores nos portadores de câncer de laringe. 
A busca por melhoria nos serviços de saúde pode auxiliar no diagnóstico precoce das lesões e no tratamento dos tumores na fase inicial, o que causaria menos desconfortos aos pacientes e melhoria da qualidade de vida dos mesmos.

Tabela 4. Resultados do questionário UW-QOL (4 versão)

\begin{tabular}{r|cccc}
\multicolumn{1}{l}{$\begin{array}{l}\text { UW-QOL } \\
\text { 12 domínios }\end{array}$} & Boca & Faringe & Laringe & Sig. \\
\hline Dor & 53,13 & 69,44 & 75,00 &, 096 \\
Aparência & 77,08 & 75,00 & 88,64 &, 275 \\
Atividade & 78,13 & 83,33 & 68,18 &, 538 \\
Recreação & 68,75 & 66,67 & 63,64 &, 905 \\
Deglutição & 59,75 & 66,56 & 81,20 &, 157 \\
Mastigação & 35,42 & 61,11 & 81,82 &, 005 \\
Fala & 75,08 & 85,33 & 63,64 &, 324 \\
Ombro & 80,54 & 85,11 & 84,82 &, 917 \\
Paladar & 62,46 & 88,89 & 63,76 &, 223 \\
Saliva & 87,54 & 88,89 & 81,82 &, 787 \\
Humor & 61,46 & 61,11 & 77,27 &, 428 \\
Ansiedade & 56,96 & 63,00 & 69,91 &, 669 \\
TOTAL & 796,29 & 894,44 & 900,27 & -
\end{tabular}

\section{REFERÊNCIAS}

ALGAVE, D.P.; MOURÃO, L.F. 2015. Qualidade de vida em laringectomizados totais: uma análise sobre diferentes instrumentos de avaliação. Rev. CEFAC. 1(1):58-70.

ANGELO, A.R.; MEDEIROS, A.C.; BIASE, R.C.C.G. 2010. Qualidade de vida em pacientes com câncer na região de cabeça e pescoço. Rev Odontol UNESP. 39(1):1-7.

BRASIL. Ministério da Saúde. 2011. Instituto Nacional de Câncer. ABC do câncer: abordagens básicas para o controle do câncer. Rio de Janeiro: Inca.

BRASIL. Ministério da Saúde. 2017. Instituto Nacional de Câncer. Estimativa 2018: incidência de câncer no Brasil. Rio de Janeiro: Inca.

GALBIATTI A.L.S. et al. 2013. Head and neck cancer: causes, prevention and treatment. Brazilian Journal of Otorhinolaryngology. 79(2).

LOPEZ, J.H. et al. 2013. Assessment of quality of life of oral cancer survivors compared with Spanish population norms. Int J Oral Maxillofac Surg. 42(4):446-452.

NEVILLE, B.W. et al. 2016. Patologia Oral e Maxilofacial. Trad. 4a Ed., Rio de Janeiro: Elsevier.

RINGASH, J.; BEZJAK A. 2001. A structured review of quality of life instruments for head and neck cancer patients. Head Neck. 23(3):201-213.

LIMA, M.A.G.; BARBOSA, L.N.F.; SOUGEY, E.B. 2011. Avaliação do Impacto na Qualidade de Vida em Pacientes com Câncer de Laringe. Rev SBPH. 14(1):18-40.

SOMMERFELD CE et al. 2012. Qualidade de vida em pacientes com câncer de cabeça e pescoço. Rev. Bras. Cir. Cabeça Pescoço. 41(4):172-177.

OLIVEIRA, R.L. et al. Prospective evaluation of quality of life in patients with head and neck cancer. Oral Surgery, Oral Medicine, Oral Pathology and Oral Radiology. 123(3):350-357. 